\title{
BLACKBIRD MONITORING SYSTEM Performance Analysis and Monitoring in Information Systems
}

\author{
João P. Germano \\ Vodafone Portugal, AV. D.João II, LT.1.04.01 Parque Nações 1998-017, Lisboa, Portugal \\ joao.germano@vodafone.com
}

Alberto R. Silva, Fernando M. Silva

INESC-ID R. Alves Redol, 9 1000-029, Lisboa, Portugal

alberto.silva@acm.org,fernando.silva@ist.utl.pt

Keywords: Application Monitoring, Systems Administration, Application Performance, Component, Adaptable.

Abstract: This work presents the BlackBird system, which is an analysis and monitoring service for data-intensive enterprise applications, without restrictions on the targeted architecture or employed technologies. Monitoring systems are an essential tool for the effective management of Enterprise Applications and the attainment of the demanding service level agreements imposed to these applications. However, due to the increasing complexity and diversity of these applications, adequate monitoring systems are rarely available. The BlackBird monitoring system is able to interact with these applications through different technologies employed by the Monitored Application, and able to produce Metrics regarding the application service level goals. The BlackBird architecture is composed by several Application Interface Modules, and by a central component responsible for Metrics calculation and presentation. Application Interface Modules interact with the target monitored Application in order to get performance data in a common format. These data are stored in a common repository and used for Metrics calculation and presentation. The BlackBird system can be specified through a set of pre-defined Configuration Objects, allowing it to be extensible and adaptable for applications with different architectures.

\section{INTRODUCTION}

As a provider of high technology services, Vodafone is under constant pressure to implement new technologies that will allow the diversification of the provided services and the improvement of existing services. Like in most large scale and technology based business, the Information Technologies (IT) infrastructure has become the main base of support to business processes, and many Enterprise Applications are now considered mission-critical having a direct impact on the performance goals of the entire company.

The complexity and diversity of the business rules and provided services, together with the pressure for fast implementation demand a vast portfolio of different applications. These applications can be extremely diverse, in terms of complexity, architecture, base technologies and application provider. Also, as result of the fierce competition environment, all these applications are required to constantly evolve in order to implement new business requirements and support new services. In organizations such as Vodafone the teams responsible for the operation and management of these applications are faced with the challenge of assuring the best possible quality of service and the attainment of the negotiated Service Level Agrement (SLA). For this task it is essential to have monitoring systems capable of providing a comprehensive view of the application status and the most critical components, in order to anticipate performance problems and act before there is any impact on the quality of service. The currently available monitoring systems can provide efficient monitoring on the network and device level, however, due to the complexity and diversity of the applications, these systems are unable to provide the desired monitoring on the application level.

Most of the available monitoring systems specifically target applications or technologies that have a large user base, are limited to a fixed architecture, and monitor pre-determined system parameters and expected system components. This kind of monitoring ignores all the functionality that is developed over the base application, even though, it is this added functionality that implements the business logic and produces the most relevant contribution to the delivered 
quality of service. The need for adequate monitoring applications is even more serious for applications developed in house or when the Monitored Application results from an extensive customization of a base application. In this case the only solution is to develop, also in house, the necessary monitoring systems. However, this extra development effort will certainly increase project cost and complexity and risk development delays.

The BlackBird monitoring system is intended for assisting in the effective management of the extremely diversified set of applications from the Information \& System Technologies department (DTSI) of Vodafone Portugal. It has two main features: $i$ ) can monitor an extremely diversified range of applications such as the one found at Vodafone; ii) provides complex Metrics that relate to the main application goals. The diagram in fig. 1 represents a general view of the monitoring system, it obtains data from a number of servers using different technologies, calculates metrics and presents the results to the Operators.

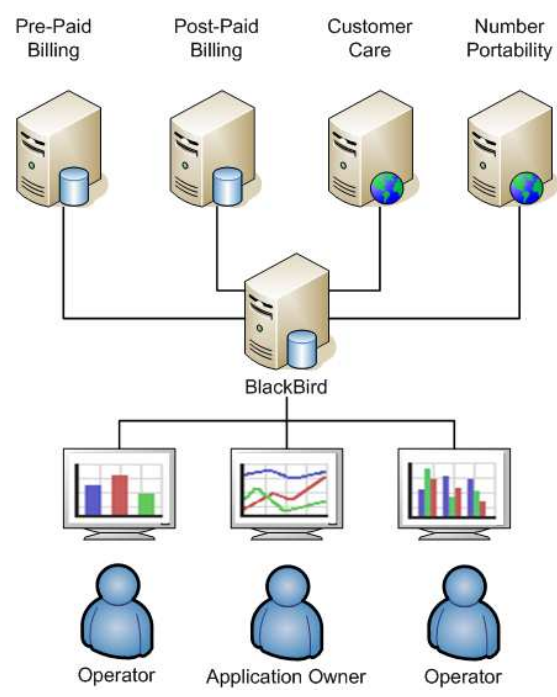

Figure 1: Monitoring System Overview.

\subsection{Existing Systems}

The need for monitoring systems was born from the need to assure high availability of the first enterprise level systems and networks, and as they matured so did the monitoring systems. Simple Network Management Protocol (SNMP) (Task-Force, 2007) became the most widely used management protocol and is currently the base to most network and device management systems, HP Openview (Hewlett-Packard, 2007) and Nagios (Galstad, 2007). However, due to the numerous programming languages and the almost infinite number of architectures and purposes, application level monitoring remains an extremely diversified field with no predominant protocols or methodologies.

Application specific systems can provide the best monitoring of any application. However, developing dedicated monitoring systems is a costly process that can only be supported by large companies with a significant application portfolio, and it becomes impossible to combine the monitoring of different applications working together. Examples of such systems are Microsoft Operations Manager (Microsoft, 2007). Third party companies will only risk developing application specific systems for applications that can guaranty a large user base. Quest Software provides versions of the Spotlight (Quest-Software, 2007) monitoring system for BEA WebLogic Server, Oracle, etc. General purpose monitoring systems aim to provide monitoring services to a range of applications as wide as possible. For this they will implement support for standard monitoring technologies and protocols, and for proprietary protocols used in applications with a large user base. The option taken by most companies for adding monitoring capabilities to their applications is to implement a standard management technology such as SNMP, JMX or WMI, for integration with a general purpose monitoring system. One example is ManageEngine (AdventNet, 2007) from AdventNet.

In terms of architecture, most monitoring systems employ a Manager-Agent architecture, there is usually a central management system and several agents deployed on the monitored system and relay the obtained data to the central management system using a protocol such as SNMP (Task-Force, 2007). This is the architecture that emerged from the first network management systems and is currently used by all monitoring systems based on the SNMP protocol. It is especially adequate for monitoring of vast numbers of distributed resources such as computer networks like Openview (Hewlett-Packard, 2007), Nagios (Galstad, 2007) or grid computing systems like MonALISA (Newman et al., 2003).

As more applications evolved from centralized to distributed and from raw processing to providing services, a new monitoring architecture became possible, Agentless Monitoring. In this case there is only a central management system, and all data gathering is accomplished by remote access to the interfaces provided by the Monitored Application. Agentless Monitoring is usually less intrusive, easier to deploy and does not require continuous development, but with limited depth of data gathering. Agentless Monitoring is especially adequate for services based 
applications and is the only option for proprietary and closed source applications. One example of agentless monitoring system is Longitude (Heroix, 2007) from Heroix.

Since the first monitoring systems one key element of the monitoring process has been application instrumentation, which consists in modifying the existing applications in order to collect additional data during run-time. The importance of application instrumentation for the management of complex distributed application resulted in several technology level standards like JMX for Java and Java2 Platform Enterprise Edition (J2EE) and WMI for Microsoft Products and the Microsoft .NET framework.

\subsection{Future Trends}

Extensive research has been aimed at improving the monitoring support of Enterprise Applications, with the main focus on improving the monitoring extensions provided by the monitored application. Most of this work is focused on the JMX (Kreger, 2001) technology which is part of J2SE platform. Although it is only applicable to Java and the J2SE platform, it has a close resemblance to the WMI for the .NET framework, and the main concepts are applicable to other programming languages and architectures, including legacy applications (Diakov et al., 2000). The use of standard management architectures and instrumentation techniques on enterprise applications opens the way to automated application management and self managed applications (Diaconescu et al., 2004). All this research presents a common characteristic, it targets component based applications, and as a result it is possible to obtain a good detail of application monitoring.

Whatever the purpose or technology used the complexity of Enterprise Applications dictates that the full task to be executed must be split between simpler tasks, that will be performed by different program modules. By considering a definition of Component less restrictive than the one usually associated with Component Based Software it should be possible to model any application as component based, where each component may have a number of parameters that can be used as indicators of the general application health and performance. And, by modeling the application, it should be possible to capture a more abstract level of application functionality, which is closer to the business logic and to the main application goals of quality of service.

\section{REQUIREMENTS}

The BlackBird monitoring system must provide five key features: $i$ ) monitor a wide range of applications, being adaptable to the architecture and technologies of the Monitored Application; $i$ ) provide in depth application level monitoring, component based; iii) easily adaptable to the evolution of the Monitored Application; iv) low impact on the monitored system, agentless and without additional application instrumentation; $v$ ) graphic interface for data visualization and configuration.

The Monitoring Systems referenced in the previous section are some of the most widely used and present a representative sample of the existing monitoring solutions. The table 1 summarizes the main features of these systems. Most of these systems allow user defined data gathering, however, the user is usually required to supply an extensive set scripts for obtaining data. The BlackBird System requires only a minimum of information for executing the same command, handles all data validation and conversion. Also, the BlackBird System allows the simultaneous execution of any commands regarded as necessary. Although most of these system provide some form of support to user defined Metrics, Metrics based on different data sources are usually limited to reporting purposes. The BlackBird System is able to provide real time Metrics based on any combination of data sources. From these systems, the ones that provide a Component Based Monitoring, support only applications developed using the frameworks J2EE or .NET. The BlackBird System introduces a simplified Component definition for extending the concept of component based monitoring to applications that were not developed as Component Base Applications.

The Blackbird System aims to provide a monitoring service to an application without imposing any limitations on the target architecture, therefore, the Blackbird System provides a simplified set of Monitoring Operations that allow a user with detailed knowledge of the Monitored Application to specify the required monitoring service: Commands to be executed where the result will be stored and used to calculate Metrics; Metrics defined by a formula to be executed on the stored data to produce a result that is related to the application's performance indicators; Alerts for evaluating thresholds on Metrics and send notifications; Graphics that use the Metric as a data source and plot the data according to the type and format; Pages for containing graphics and structuring the monitoring interface. 
Table 1: Feature Comparison Table.

\begin{tabular}{|c|c|c|c|c|c|c|c|}
\hline & $\begin{array}{l}\text { Targeted } \\
\text { Systems }\end{array}$ & $\begin{array}{l}\text { Supported } \\
\text { Protocols }\end{array}$ & $\begin{array}{l}\text { Agent } \\
\text { Based }\end{array}$ & Interface & $\begin{array}{c}\text { User Defined } \\
\text { Data Gathering }\end{array}$ & $\begin{array}{c}\text { User Defined } \\
\text { Metrics }\end{array}$ & $\begin{array}{c}\text { Component } \\
\text { Based }\end{array}$ \\
\hline $\begin{array}{l}\text { HP Openview } \\
\text { Operations }\end{array}$ & $\begin{array}{l}\text { General } \\
\text { Purpose }\end{array}$ & SNMP & $\mathrm{Y}$ & $\begin{array}{l}\text { Thin } \\
\text { Client }\end{array}$ & $\mathrm{Y}$ & $\mathrm{Y}$ & $\mathrm{N}$ \\
\hline Nagios & $\begin{array}{l}\text { Network } \\
\text { and Host }\end{array}$ & Network services & Optional & Web & $\mathrm{Y}$ & $\mathrm{Y}$ & $\mathrm{N}$ \\
\hline $\begin{array}{c}\text { Microsoft } \\
\text { Operations Manager }\end{array}$ & $\begin{array}{c}\text { Microsoft } \\
\text { Applications }\end{array}$ & WMI & $\mathrm{Y}$ & $\begin{array}{c}\text { Web } \\
\text { and Client }\end{array}$ & $\mathrm{Y}$ & $\mathrm{Y}$ & $\mathrm{Y}$ \\
\hline Spotlight & Dedicated & JMX & $\mathrm{Y}$ & $\begin{array}{c}\text { Thin } \\
\text { Client }\end{array}$ & $\mathrm{N}$ & $\mathrm{N}$ & $\mathrm{Y}$ \\
\hline Manage Engine & $\begin{array}{l}\text { General } \\
\text { Purpose }\end{array}$ & Multiple & Optional & Web & $\mathrm{Y}$ & $\mathrm{N}$ & $\mathrm{Y}$ \\
\hline Longitude & $\begin{array}{l}\text { General } \\
\text { Purpose }\end{array}$ & Multiple & $\mathrm{N}$ & Web & $\mathrm{Y}$ & $\mathrm{Y}$ & $\mathrm{Y}$ \\
\hline BlackBird & $\begin{array}{l}\text { General } \\
\text { Purpose }\end{array}$ & Multiple & $\mathrm{N}$ & Web & $\mathrm{Y}$ & $\mathrm{Y}$ & $\mathrm{Y}$ \\
\hline
\end{tabular}

\section{ARCHITECTURE}

As proposed in section 1.2 , by modeling the Monitored Application as a set of interacting components it should be possible to obtain a more de detailed view of the application status and performance, also, it should be easier to obtain Metrics that relate to the application performance goals. For the purposes of this work, we consider a simplified definition of Application Component: i) executes a well defined task within the application; ii) can be univocally referenced; iii) has a set of working parameters which can be obtained using the application interfaces. Figure 2 represents the Monitored Application according to this definition.

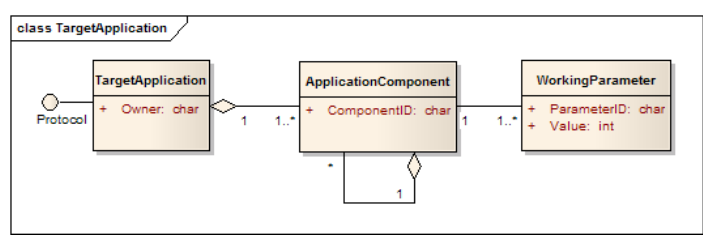

Figure 2: Application Model.

Figure 3 presents the high level Domain Model for the BlackBird System. Each of the required Monitoring Operations is implemented by a dedicated class, except for Commands that is split between the Module class and subclasses dedicated to specific technologies, DatabaseScript and WebserviceRequest. The dataStore attribute of Module stores all data produced by the associated command and provide support to Metrics calculation.

The Module sub classes provide an adaptation layer that isolates the BlackBird architecture from any technology details, they handle all technology spe-

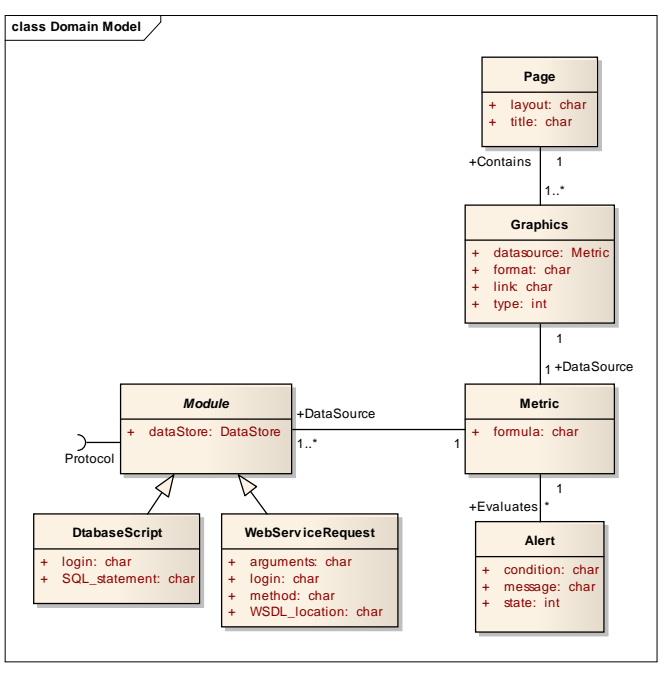

Figure 3: Domain Model.

cific logic like establishing a connection, authentication, formatting the command, obtaining and validating the response. Finally, they convert the command result to a normalized Extensible Markup Language (XML) document and deliver that document to the dataStore. For simplifying Metrics definition and calculation, the dataStore is designed to be accessed as a relational entity. The Metric class provides the data processing and aggregation functionalities of the BlackBird System by computing the formula specified in the Monitoring Requirement. The Graphic class provides the visual presentation to the Metric objects, it will use the output of the Metric as a data source and apply the type of graphic and the format request in the Monitoring Requirement. A Graphic may be a table of values or various chart formats. The Page class provides the base for generating the mon- 
itoring interface that will be accessed and navigated by the operators. By combining the information from the Page and Graphic objects, the BlackBird system generates the required interface as an Web Applicaton containing the requested charts and tables. The Alert class provide automatic notification of performance problems. It is defined as boolean condition to be evaluated.

\subsection{BlackBird Components}

The Blackbird system uses an agentless architecture, it is composed of an Adaptation Layer itself composed by a variable number local Interface Modules designed for specific protocols, an Aggregation Layer that handles data storage and Metrics calculation, and a Presentation Layer for generating the monitoring pages and graphics. The Component Diagram of the BlackBird System is represented in fig. 4.

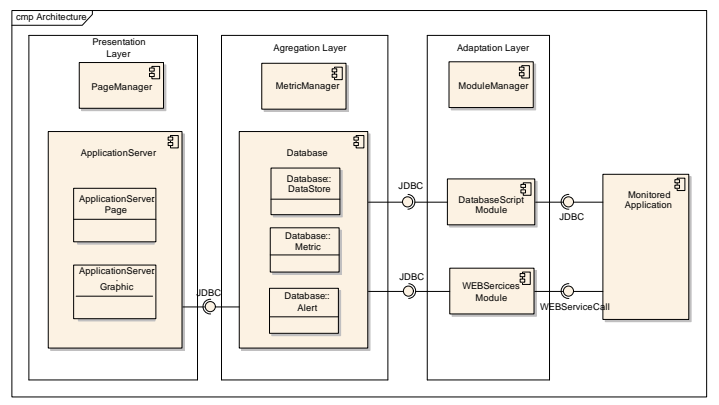

Figure 4: BlackBird Components.

The Adaptation Layer provides one of the main features of the BlackBird System, adaptation to the technologies of the Monitored Application. All technology and protocol specific processing is performed by interface modules, where each type of interface module handles a specific technology or protocol, performs all the tasks necessary for executing the requested command, converts the command output to the normalized format and delivers it to the dataStore . The Adaptation Layer also performs the first step for providing a monitoring service adapted to the architecture of the monitored system. By allowing multiple Module to execute independently it becomes possible to specify as many data sources as required for compiling a complete repository of performance data that will allow the calculation of any relevant Metrics. The ModuleManager component controls execution of the Interface Modules and manages the dataStore of those objects. For this work only two modules where developed, an SQL module that uses JDBC to connect to the monitored Database for executing an SQL statement and an Web Services module that implements the Apache Axis Framework for dynamically invoking Web services. These technologies are base to most of Vodafone applications, and allow the demonstration of the BlackBird capabilities in combining data obtained using different technologies.

The BlackBird architecture is expandable to other types of protocols. Adding support for aditional protocols to the BlackBird system requires no changes to existing components only the development of: $i$ ) a new interface module to handle the required protocol; ii) two new configuration tables and associated views; iii) new stored procedures for the edit operations on the new type of module.

The Aggregation Layer stores all performance data in an organized an easily accessible form, performs Metrics calculation and alerts verification. The Aggregation Layer contains the dataStore attributes from all existing Module objects implemented as database tables and views, the combination of all these objects constitutes a complete repository of all performance data gathered from the Monitored Application. And, since all this data is accessible through relational queries, it should be possible to implement any Metric required by the Application Owner. The Aggregation Layer is responsible for other of the main features of the BlackBird System, Component Based Monitoring. Since all performance data gathered from the application can be used as input for the Metric objects, it is possible aggregate the data collected by different Modules using the component identifier to produce Metrics that provide a complete view of all aspects for that Application Component. The MetricManager component is responsible for creating and updating the implementation of Metric formulas, and actively evaluating alert conditions.

The Presentation Layer is responsible for the final output of the BlackBird Monitoring System, which are monitoring pages containing visualizations of the status and performance of the Monitored Application. The monitoring pages are generated from the Graphic and Page objects and deployed on an Application Server as a Web Application. However, the automated generation of the Web Application was considered to be out the scope of this work, so the Webcockpit (Klauser, 2007) application is used to generate the monitoring pages based on a configuration file created by the BlackBird System from the existing Page and Graphic objects. The PageManager component is responsible for managing the generation and deployment of the Web Application that provides the monitoring interface. 


\section{IMPLEMENTATION AND DESIGN}

In order to isolate the BlackBird architecture from any technology details it is necessary to define a normalized format for the results data, this format must be: $i$ ) capable of containing any result; $i$ ) easily convertible to a relational format. According to the application model defined in the previous section (fig. 2), and to support component level monitoring, the output of a command executed on the monitored system may be defined as a tree of components each containing any number of working parameters, including a component identifier. According to this definition, any result may be formatted as a XML document with the schema from fig. 5 .

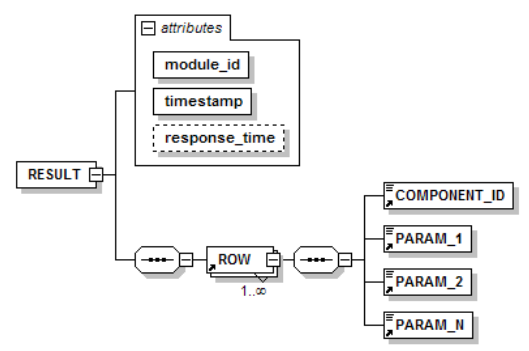

Figure 5: XML Schema.

Because the BlackBird System is intended for an enterprise environment where security and role separation is always a major concern, for each Monitored Application there is a dedicated database schema for contain the implementations of dataStore Metric and Alert for that application. The BlackBird main schema presented in fig. 6 is used for controlling the monitoring process, contains Monitoring Objects definitions, and additional entities for implementing application separation and access control. The Monitored Application Schema contains the database objects that implements the dataStore and Metric. Figure 7 presents an example schema containing the implementation of two dataStore and two Metric objects.

The final implementation of datastore is composed of a Results Table and a Translation View, result data is stored XML format in the Results Table, then based on the format of the XML result the BlackBird System creates a view for selecting all XML elements as columns of result set. The complex implementation of the datastore allows the implementation of the Metric object simply as a database view created from the SQL formula defined in the Metric .

The final output of the monitoring process is an Web application containing charts and tables that pro-

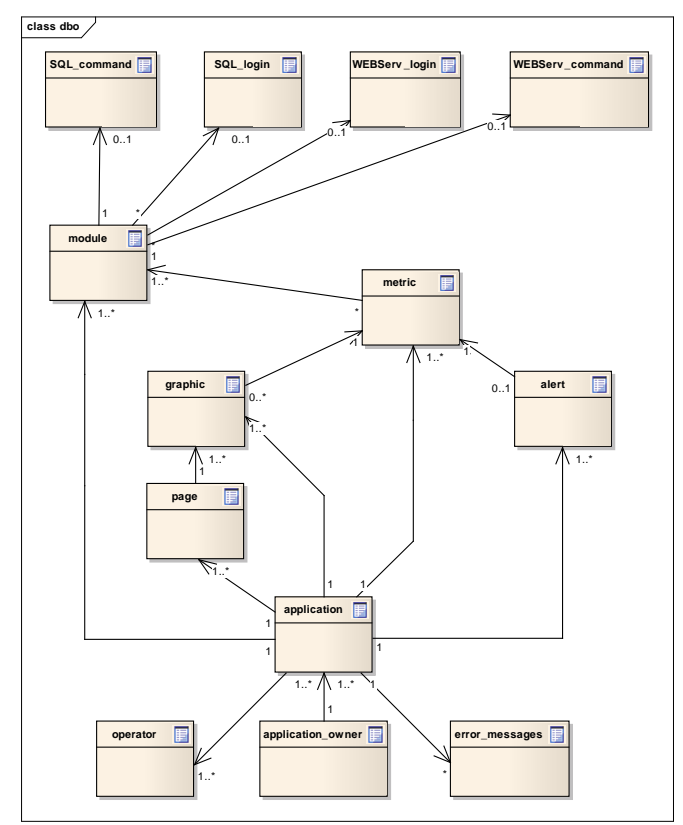

Figure 6: Main Schema.

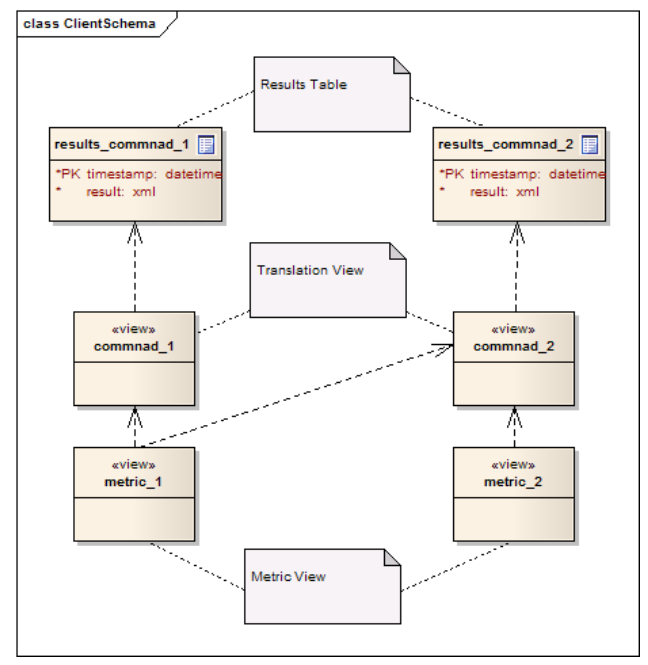

Figure 7: Monitored Application Schema.

vide a visual representation of Metrics. From the existing Page and Graphic objects, the PageManager generates the Webcockpit configuration file that specifies the contents of the required Web application. Then, the Webcockpit application is used to generate the Java Server Pages (JSP) that implement the required charts and tables.

\section{CASE STUDY}

The BlackBird System is currently integrated in the production environment of some of the main appli- 
cations of Vodafone Portugal DTSI, Mediation Device (MD), Pre Paid Billing System (PPB), ARBOR Billing System, Provisioning Agent (PA) and ACTIVIS Number Portability. All are Mission Critical applications that require 24 hour support and monitoring. The previously existing monitoring systems, HP Openview Operations and dedicated monitoring systems, provide complete network and device monitoring and a small degree of application level monitoring. The BlackBird System is used to complement these systems providing detailed application level monitoring.

\subsubsection{Pre Paid Billing System}

The PPB application is responsible for lifecycle management of all pre-paid clients, it was developed at Vodafone, it contains an Oracle database and was developed using $\mathrm{C}$ and PL/SQL. This example will add monitoring of recent functionalities of the PPB application that have not yet been included in the dedicated monitoring system.

Recent Business Requirements have introduced new types of requests, a database table acts as processing queue and the processing of each request requires a call to a WebService for confirming current balance. The main performance criteria for this task are the average processing time and maximum processing time for these requests. For monitoring this aspect of the PPB application, problems diagnosis and performance tuning, it would be extremely helpful to have a line chart presenting the average request execution time, the average Web Service response time, and the difference between the two times, which represents the contribution of the PPB System to the total processing time. This is a relatively simple example, however, the presented parameters have direct relation to the application performance goal. Furthermore, by splitting the application goal into sub parameters, it becomes easier to identify the contribution of each application component to the final result.

Once the required output is defined, the Application Owner must elaborate the Monitoring Requirement in order to specify the Configuration Objects necessary for producing the desired monitoring output. The Monitoring Requirement for this example contains the following Configuration Objects: $i$ ) Login details for the PPB database; ii) Login details for Web Service calls; iii) SQL command for obtaining the average processing time; $i v)$ Web command for obtaining the Web Service response time; v) Metric for combining data from both commands; vi) A line chart that uses that Metric ; vii) A page for containing the the Graphic .

The final output of the PPB monitoring example is shown in fig. 8. This chart shows the evolution of the

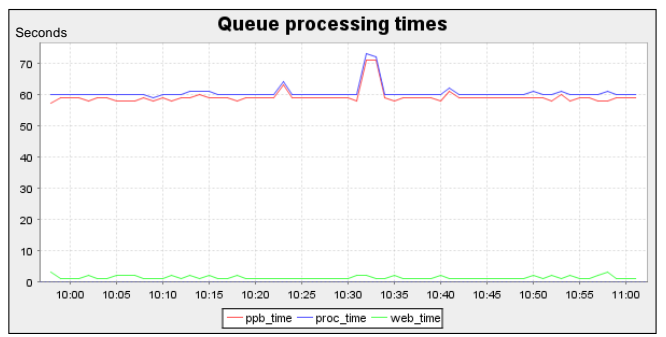

Figure 8: PPB Queue.

requests processing time and the contributions of the Web Service call and PPB processing.

In this example the BlackBird System was used for fast implementation of a detailed monitoring service, and to generate Metrics that aggregate data obtained using different technologies, Database access and Web Services call.

\subsubsection{Mediation Device}

The Mediation Device processes billing records of all traffic types, GSM, 3G, GPRS, SMS, etc. It collects billing records from all Network Elements, validates and rates billable records. The Mediation Device was also developed at Vodafone, it contains an Sybase database and was developed using $\mathrm{C}$ and Transact SQL. The Mediation Device processes various types of records refereed to as Data Stream, and for each Data Stream, there are various Network Elements, this architecture can easily be modeled into components. The basic components are the Network Elements that have parameters such as current delay and records processing rate. These elements can be grouped into the main application components the Data Streams. The most critical SLA defined for the Mediation Device are all related to the delay between record generation on the network element and its delivery to the destination billing systems, and the fundamental indicator of performance is the number of records processed per unit of time.

The first chart from fig. 9 shows the evolution of the delay in Call Records processing for the Network Element components of the MD application. The second char on fig. 9 also shows the evolution of number of Call Records processed per hour for the Stream components of the MD.

In this example the BlackBird System was used to improve the existing monitoring to detail the information on the component level, and provide real time visualization of Metrics directly related to the SLA an performance indicators. 


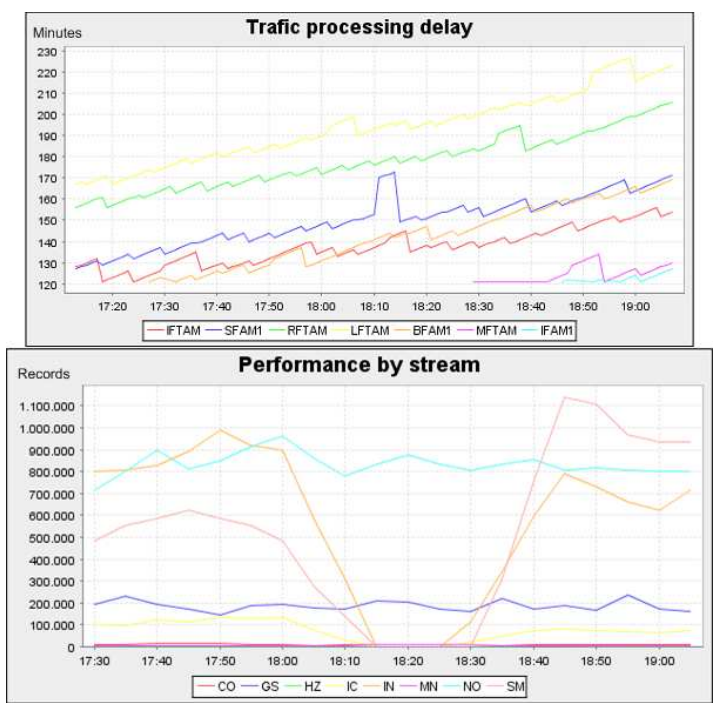

Figure 9: Mediation Device Streams.

\section{CONCLUSIONS AND FUTURE WORK}

The BlackBird System aims to provide detailed application level monitoring to a range of applications as wide as possible, requiring a minimum of configuration work. The use of a simplified application model creates a language for describing most applications as a component hierarchy. Also, it allows the definition of common data format for containing performance information independently of any technology details. Data gathering is performed by technology specific Interface Modules, where the Application Owner may specify any number of modules for various protocols. This way, it becomes possible to gather any relevant data independently of the target architecture or the employed technologies. The performance data is stored in DataStore units accessible as relational entities. Data can be referenced by specifying the Module that collected the data and the application component it refers to. Based on this data repository it is possible to create complex metrics that merge data from multiple sources, and metrics that relate to the performance goals for the application.

Although the data repository provides an easily accessible source of data, it can become very complex as the number Interface Modules increases. Also, the task of defining Metrics in the BlackBird system requires a good knowledge of the BlackBird architecture and SQL. This task can be greatly simplified by the addition of a graphical query building interface, as an applet on the Configuration Pages or as a separate application.
The BlackBird was designed as an expandable System, therefore some of the main improvements will be new Interface Modules for additional protocols: Remote Shell for access to unix systems, JMX for J2EE applications, WMI for .NET application.

\section{REFERENCES}

AdventNet (2007). Applications Manager. http://manageengine.adventnet.com/products/ index.html.

Diaconescu, A., Mos, A., and Murphy, J. (2004). Automatic performance management in component based software systems. Proceedings of the International Conference on Autonomic Computing.

Diakov, N. K., van Sinderen, M., and Quartel, D. (2000). Monitoring extensions for component-based distributed software. Fifth International Conference on Protocols for Multimedia Systems.

Galstad, E. (2007). Nagios 3.x documentation. http://nagios.sourceforge.net/docs/nagios-3.pdf.

Heroix (2007). Longitude. http://www.heroix.com/ agentless/agentless_performance_monitoring_main.htm.

Hewlett-Packard (2007). HP Operations Dashboard software. http://www.managementsoftware.hp.com/ products/ovd/ds/ovd_ds.pdf.

Klauser, P. J. (2007). Webcockpit Open Source Project. http://webcockpit.sourceforge.net/schema/ schema.html.

Kreger, H. (2001). Java management extensions for application management. IBM SYSTEMS JOURNAL, 40.

Microsoft (2007). System Center Operations Manager. http://technet.microsoft.com/enus/opsmgr/default.aspx.

Newman, H., I.C.Legrand, Galvez, P., Voicu, R., and Cirstoiu, C. (2003). Monalisa : A distributed monitoring service architecture. $C H E P 03$.

Quest-Software (2007). Spotlight for BEA WebLogic Server. http://www.quest.com/spotlight-for-beaweblogic-server/.

Task-Force (2007). Simple network management protocol (snmp) applications. ftp://ftp.rfc-editor.org/innotes/rfc3413.txt. 